\title{
Diferença em nível de stress em duas amostras: capital e interior do estado de São Paulo
}

\author{
The stress level difference between the country \\ and the capital in São Paulo state
}

\author{
Eliana Aparecida Torrezan da SILVA \\ Alessandra MARTINEZ
}

\begin{abstract}
Resumo
Este estudo avaliou o nível de stress e sua sintomatologia em pessoas atendidas em um serviço oferecido à comunidade. A amostra foi composta de 126 pessoas de ambos os sexos divididas em dois grupos: 62 moradores do interior e 64 da capital, São Paulo. Os resultados mostraram que $79 \%$ do total da amostra apresentaram sintomas significativos do stress e que não houve diferença significativa entre interior e capital. Os sintomas psicológicos foram os mais detectados entre os participantes. Esses dados sugerem a elaboração de programas, não somente de profilaxia, mas de tratamento do stress específico para a comunidade.
\end{abstract}

Palavras-chave: comunidade; inventário de stress de Lipp; stress.

\begin{abstract}
This paper has evaluated the stress level and its symptomatology in people attended by the public service department. One hundred twenty-six people were examined and divided into two groups: 62 from the country and 64 from the capital. The results has shown that 79\% presented significant stress symptoms without regional distinction, and the psychological symptoms incidence was higher than the others. According to these data, prophylactic and stress treatment programs should be developed focused on the community needs.
\end{abstract}

Key words: community; Lipp's Stress Inventary; stress.

A palavra stress, de origem latina, vem do verbo "stringo", que significa apertar, cerrar, comprimir, espreitar, restringir, diminuir e reduzir, de acordo com o dicionário Webster's (1950). No século XVII, o termo passou a ser utilizado para designar angústia, opressão, injustiça e adversidade. Posteriormente, nos séculos XVIII e XIX, o vocábulo foi usado para denotar opressão ou uma forte influência atuando sobre um objeto físico ou uma pessoa (Spielberger, 1979). O termo foi mencionado pela primeira vez na área da saúde em 1925, por Selye, um estudante de medicina que analisou respostas normais e patológicas dos pacientes atendidos nos ambulatórios. Após realizar muitos estudos experimentais, denominou de "Síndrome de Adaptação Geral" a somatória das reações corporais resultantes da exposição às fontes de stress (Selye, 1965).

$\boldsymbol{\nabla v} \mathbf{v}$

1 Faculdade de Psicologia Unicastelo. Rua Carolina Fonseca, 235, 08230-030, São Paulo, Brasil. Correspondência para/Correspondence to: E.A.T. SILVA. E-mail: <etorrezan@zipmail.com.br>. 
As reações do stress podem ser manifestadas em nível físico e/ou psicológico. De acordo com Lipp (1984), as manifestações físicas podem ser aumento da sudorese, hiperacidez estomacal, tensão muscular, taquicardia, hipertensão arterial, bruxismo e náuseas. As manifestações psicológicas podem ser ansiedade, angústia, dúvidas quanto a si próprio, dificuldade de concentração, preocupação excessiva e hipersensibilidade excessiva.

A reação do stress pode ser dividida em fases, de acordo com Selye (1965): alerta, resistência e exaustão. Lipp (2000) acrescentou uma nova fase quase-exaustão, que seria a fase intermediária entre a resistência e a exaustão. As fases estão descritas a seguir:

1) Fase de alerta: quando o organismo é exposto a uma situação produtora de tensão, ele se prepara para a ação, através da mobilização de alterações bioquímicas, compreendendo as reações de "luta e fuga" definidas por Cannon (1953). Algumas reações presentes são taquicardia, tensão muscular e sudorese. Quando o agente estressor - o que está gerando o stress - não é eliminado, o organismo passa ao estágio de resistência.

2) Fase de resistência: nessa fase, o indivíduo, automaticamente, utiliza energia adaptativa para se reequilibrar. Quando consegue, os sinais iniciais (das reações bioquímicas) desaparecem e o indivíduo tem a impressão de que melhorou. A sensação de desgaste generalizado, sem causa aparente, e as dificuldades com a memória ocorrem nesse estágio, mas, muitas vezes, não são identificadas pelo indivíduo em situações de stress excessivo.

3) Fase de quase-exaustão: o organismo está enfraquecido e não consegue se adaptar ou resistir ao estressor. As doenças começam a aparecer, tais como herpes simples, psoríase, picos de hipertensão e diabete, nos indivíduos geneticamente predispostos (Lipp, 2000). Quando o estressor permanece atuante por muito tempo, ou quando muitas fontes de stress ocorrem simultaneamente, a reação do organismo progride para a fase de exaustão.

4) Fase de exaustão: observa-se nessa fase que há um aumento das estruturas linfáticas, a exaustão psicológica e a física se manifestam e em alguns casos a morte pode ocorrer. As doenças aparecem com muita 54 freqüência tanto em nível psicológico, em forma de depressão, ansiedade aguda, inabilidade de tomar decisões, vontade de fugir de tudo, como também em nível físico, com alterações orgânicas, hipertensão arterial essencial, úlcera gástrica, psoríase, vitiligo e diabete. Convém observar que o stress não é o elemento patogênico das doenças, ele conduz a um enfraquecimento do somático e do psicológico de tal modo que aquelas patologias programadas geneticamente se manifestam devido ao estado de exaustão presente (Lipp \& Malagris, 1995).

Lipp, Romano, Covolan e Silva (1990) mencionam que o stress promove um desgaste geral do organismo, que é causado pelas alterações psicofisiológicas que ocorrem quando a pessoa se vê forçada a enfrentar uma situação estressante. Witkin-Lanoil (1985), Torrezan (1997) e Lipp (2003) concordam que o stress excessivo pode desencadear ou potencializar doenças, tais como gastrite, úlcera, hipertensão arterial, herpes simples, dermatites entre outras.

Nas várias definições do termo stress encontradas na literatura, como, por exemplo, em Selye (1965), Everly e Rosenfeld (1981), Lipp (1984), Witkin-Lanoil (1985), Lipp e Malagris (1995), Molina (1996) e CID - 10 (1997), observa-se que há coerência sobre seu conceito, uma vez que todas as explicações apontam para a necessidade do corpo se adaptar a certas situações de tensão, e sobre o processo que envolve alterações psicofisiológicas.

Lipp (2003) menciona que o stress é uma reação do organismo, com componentes físicos e/ou psicológicos que ocorrem quando a pessoa se confronta com uma situação que, de um modo ou de outro, a irrite, amedronte, excite ou confunda, ou mesmo que a faça imensamente feliz.

O que pode ser verificado é que não somente as situações consideradas ruins, mas também as boas desencadeiam as reações de stress. Segundo Molina (1996), o stress pode ser definido como qualquer situação de tensão aguda ou crônica que produza uma mudança no comportamento físico, no estado emocional do indivíduo, ocorrendo uma resposta de adaptação psicofisiológica que pode ser negativa ou positiva para o organismo. Desse modo, a área fisiológica e a comportamental da pessoa são afetadas. Essa reação de stress também está inserida na 
Classificação Internacional das Doenças (CID - 10, 1997). O CID - 10 inclui o stress na categoria (F-43) reações ao stress e transtornos de adaptação.

Nota-se que a ocorrência do stress varia dependendo de quanto o indivíduo foi afetado. Um pouco de stress desencadeia um movimento do organismo para ação, de modo que haja energia para lidar com as situações. Contudo, quando o stress atua por um longo período de tempo, ou é muito intenso, o organismo tem que despender muita energia, provocando um desequilíbrio, podendo assim se tornar vulnerável às doenças.

Nos tempos modernos, juntamente com o avanço tecnológico, também surgiram problemas que acompanham as transformações. Um local que poderia ser referendado com uma boa qualidade de vida e pouco stress muitas vezes pode não ser. As cidades do interior de um Estado talvez possam ou ofereçam condições de vida que proporcionem menos stress. Pode-se dizer que o fato de morar na capital, com um número maior de habitantes, problemas específicos dessa demanda, como insegurança, desemprego, falta de saneamento básico, falta de assistência à saúde entre outros, talvez acabe gerando stress na busca em suprir as necessidades básicas. Pode-se, então, pensar que a região, as crenças, os hábitos, a cultura de um país possam interferir na resposta de stress. Considerando a localização regional no país e a incidência de stress em determinada ocupação, o estudo de Baechtold (2002) com costureiras na cidade de Blumenau mostrou que a incidência do stress esteve presente em $86 \%$ da amostra, o que mostra que o stress pode estar presente, independentemente da cultura e localização geográfica, em diversas partes da sociedade brasileira. A pesquisa realizada por Lipp, Pereira, Floksztrumpf, Muniz e Ismael (1996) verificou o nível de stress entre homens e mulheres na cidade de São Paulo. Participaram desse estudo 1818 pessoas de ambos os sexos, sendo que 32\% apresentaram sintomas significativos do stress. Encontrou-se, também, uma diferença significativa entre homens e mulheres: as mulheres apresentaram mais sintomas de stress que os homens. Os dados mostraram que o stress estava elevado nessa população.

Um outro estudo realizado por Areias e Guimarães (2004) investigou o gênero e o stress em trabalhadores de uma universidade pública do Estado de São Paulo. Os resultados mostraram associações significativas entre saúde mental e gênero e entre fatores psicossociais de risco e gênero. Participantes do sexo feminino apresentaram maiores fatores de risco, stress no trabalho, stress social e pior saúde mental que os do sexo masculino, com maior risco de adoecimento físico e/ou mental.

Considerando a importância de estudar cada vez mais os efeitos e prejuízos do stress excessivo para o ser humano, o presente estudo objetivou identificar a incidência do stress em moradores de duas localidades: uma cidade do interior do Estado de São Paulo e a capital do mesmo estado.

\section{Método}

A amostra foi composta de 126 pessoas, sendo 62 avaliadas em uma cidade do interior do Estado de São Paulo e 64 avaliadas na capital do estado. Essas pessoas participaram de um projeto comunitário - promovido por uma universidade particular - que tem como objetivo oferecer gratuitamente diversos serviços à comunidade, tais como: orientação psicológica, odontológica, prática física, cuidados com a saúde, documentação, entre outros.

Em relação à idade dos entrevistados, verificou-se que no interior a idade variou de 20 a 57 anos, sendo a média de 34 anos, e na capital a idade variou de 16 a 65 anos, sendo a média de 29 anos. A idade média da amostra é de 30 anos.

Quanto ao gênero dos participantes, no interior, $84 \%$ eram do sexo feminino e $16 \%$ do sexo masculino. Na capital, os entrevistados eram $73 \%$ do sexo feminino e $27 \%$ do sexo masculino.

A escolaridade dos entrevistados no interior distribuiu-se da seguinte forma: $32 \%$ com ensino fundamental incompleto, $16 \%$ com ensino fundamental completo, 5\% com ensino médio incompleto, 16\% ensino médio completo, 16\% com nível superior incompleto, 13\% com nível superior completo e 2\% com pós-graduação. Na capital, verificou-se que 14\% dos participantes possuíam ensino fundamental incompleto, 9\% ensino fundamental completo, 16\% ensino médio incompleto, $11 \%$ ensino médio 
completo, $27 \%$ nível superior incompleto e $23 \%$ dos participantes possuíam nível superior completo. Nota-se que o nível de escolaridade dos participantes do interior era, em sua maioria, do ensino fundamental, e o dos entrevistados da capital, em sua maioria, era nível superior. Esse dado mostra que existe uma diferença na prevalência dos níveis de escolaridade entre os grupos.

No que se refere à profissão dos participantes da cidade do interior do estado, havia auxiliar de serviços gerais, faxineira, secretária, administrador, professora, auxiliar de biblioteca, gerente, balconista, servente de pedreiro, vigia, inspetor de alunos, motorista, auxiliar de enfermagem, investigador, doméstica, do lar, estudante e alguns se encontravam desempregados. Na capital, as profissões dos entrevistados compreenderam as seguintes funções: professor, auxiliar administrativo, auxiliar de enfermagem, analista contábil, pedreiro, estagiário de direito, técnico em informática, chefe de departamento, profissional autônomo, auxiliar mecânico, empregada doméstica, do lar, auxiliar geral, bancário, secretária, estudantes e desempregados. Observa-se que as profissões dos participantes foram variadas, não havendo uma prevalência em determinada função.

Os instrumentos utilizados na pesquisa foram: 1) Formulário de identificação: que propõe identificar a idade, o sexo, o nível de escolaridade e a profissão dos entrevistados. 2) Inventário de Sintomas de Stress Adulto (ISSL) (Lipp, 2000), que identifica o nível de stress e sua sintomatologia, avaliando se a pessoa possui sintomas de stress, a natureza dos mesmos (se somáticos ou psicológicos) e a fase de stress em que se encontra. O ISSL é composto de quatro partes, alerta, resistência, quase-exaustão e exaustão. Essa divisão tem base nos princípios de Selye (1965), quanto às fases do processo de stress.

As avaliações ocorreram na própria universidade, na capital e interior de São Paulo, em salas onde havia uma mesa, três cadeiras, iluminação e ventilação satisfatórias.

Durante a execução do programa comunitário, foram afixados cartazes em diversas áreas da universidade, divulgando a avaliação do nível de stress. Desse modo, as pessoas que se interessaram em participar voluntariamente da avaliação procuraram o serviço de psicologia. Na entrevista com cada sujeito era informado o objetivo do projeto e que esse era sigiloso, ou seja, a identificação dos sujeitos não seria divulgada. Após o consentimento por parte de cada participante, iniciou-se o preenchimento do formulário de identificação e aplicação do ISSL. Após a avaliação, os entrevistados receberam orientação sobre o que é stress, seus sintomas e como gerenciá-lo. As informações quanto ao stress foram descritas verbalmente e os sujeitos receberam uma versão impressa.

\section{Resultados}

Os dados foram obtidos através das avaliações que ocorreram primeiro com os moradores da cidade do interior e a seguir com os moradores da cidade de São Paulo. A avaliação ocorreu em um único dia, em ambos os locais.

A avaliação do nível de stress realizada com as 126 pessoas revelou que o stress esteve presente na amostra. Os dados foram apresentados, inicialmente, em três etapas: os dados referentes às entrevistas no interior, os dados da capital e finalmente os resultados globais.

\section{Nível de stress no interior e na capital}

Os resultados mostraram que $82 \%$ da amostra da cidade do interior apresentaram stress e 18\% não indicaram sintomas significativos. Dentre as pessoas que se encontravam estressadas, 53\% estavam na fase de resistência, 26\% na fase de quase-exaustão e 3\% na fase de exaustão (Figura 1). Observou-se, também, que $2 \%$ dos entrevistados estavam na fase de alerta. A fase de alerta pode ser considerada positiva para a produtividade do ser humano, pois é necessário um pouco de stress para se realizar tarefas. Além disso, essa fase indica que a pessoa entrou e saiu do processo de stress e não ocasionou prejuízos para a sua saúde.

As análises quanto ao nível de stress seguiram as mensurações estabelecidas pelo ISSL (Lipp, 2000).

No que se refere à avaliação do nível de stress dos participantes da capital, verificou-se que 78\% da amostra apresentaram níveis significativos do stress, 
sendo 58\% na fase de resistência, 17\% na fase de quase-exaustão e 3\% na fase de exaustão. Observou-se que 22\% dos participantes não indicaram sintomas significativos do stress. Dentre os entrevistados que não estavam estressados, compreenderam-se os que não identificaram sintomas do stress e aqueles que estavam na primeira fase do stress, a alerta (Figura 2).

Considerando os 126 participantes, observou-se que $79 \%$ apresentavam sintomas significativos de stress. O nível de stress na amostra esteve concentrado na segunda fase, a de resistência, que é considerada moderada, mas que ocasiona um desequilíbrio interno no organismo.

\section{Incidência do stress nos moradores da capital e do interior}

Não se encontrou diferença significativa entre a incidência de stress na capital e no interior. O teste Mann-Whitney mostrou que $U(0,80)$ não é estatisticamente significante, $p=0,42$. Não há diferença nos níveis de stress entre os participantes do interior e da capital.

\section{Sintomatologia do stress}

Quanto aos sintomas que fazem parte do quadro de stress, verificou-se, na cidade do interior, que os sintomas psicológicos foram os mais mencionados.

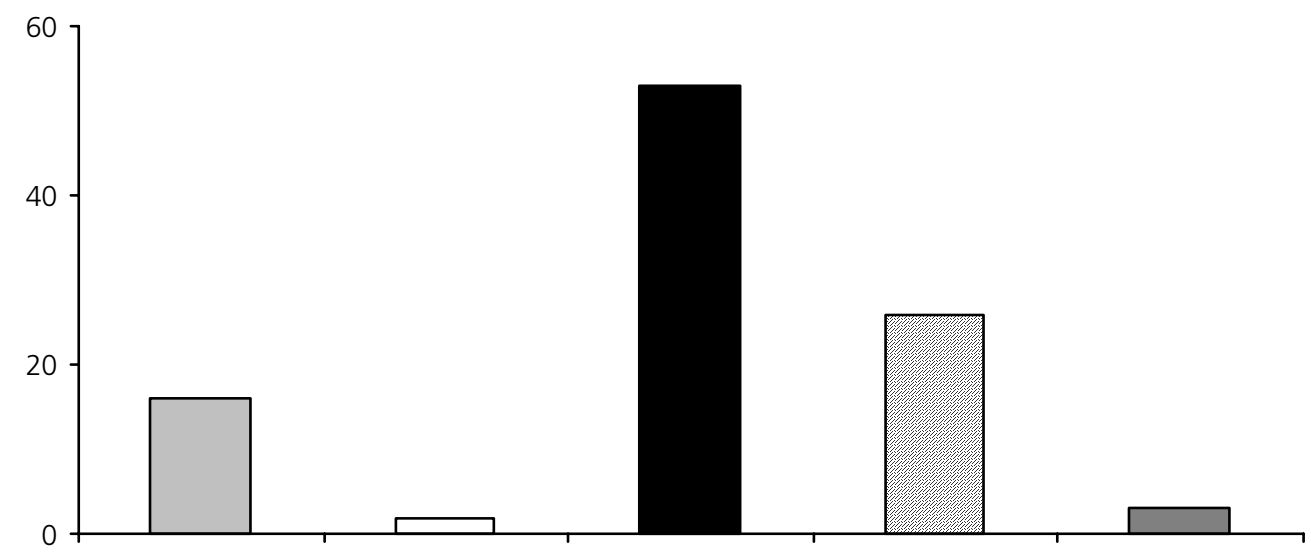

Figura 1. Distribuição do nível de stress nos participantes do interior.

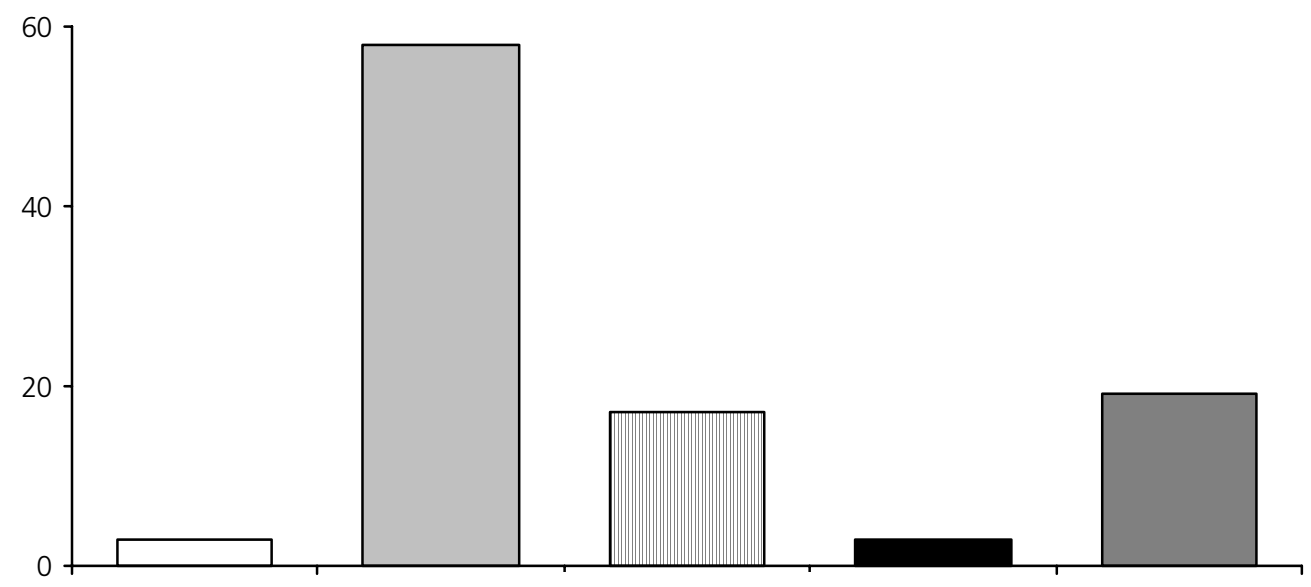

Figura 2. Distribuição do nível de stress nos participantes da capital. 
Os dados mostraram que $63 \%$ dos participantes mencionaram os psicológicos, $16 \%$ os físicos e $8 \%$ ambos, psicológicos e físicos. Verificou-se que 53\% dos participantes da capital apresentaram sintomas na área psicológica, 19\% na área física e 9\% em ambas as áreas. Os sintomas psicológicos foram os mais mencionados em ambos os grupos (Figuras 3 e 4).

\section{Sintomas mais mencionados pelos participantes}

A análise detectou que os sintomas psicológicos mais referidos pelos dois grupos foram sensibilidade emotiva excessiva, pensar constantemente em um só assunto e dúvida quanto a si próprio. Os sintomas físicos

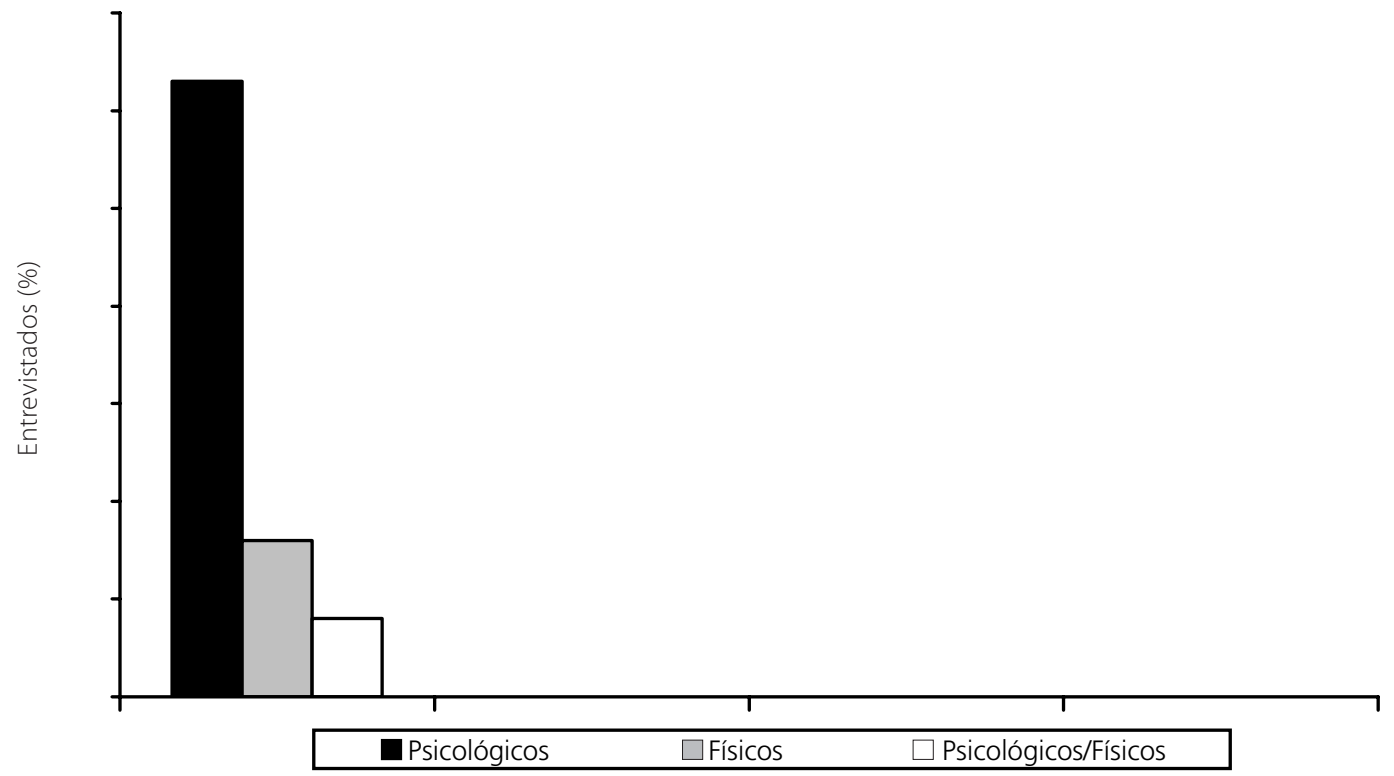

Figura 3. Distribuição da incidência de sintomas do stress entre os entrevistados do interior.

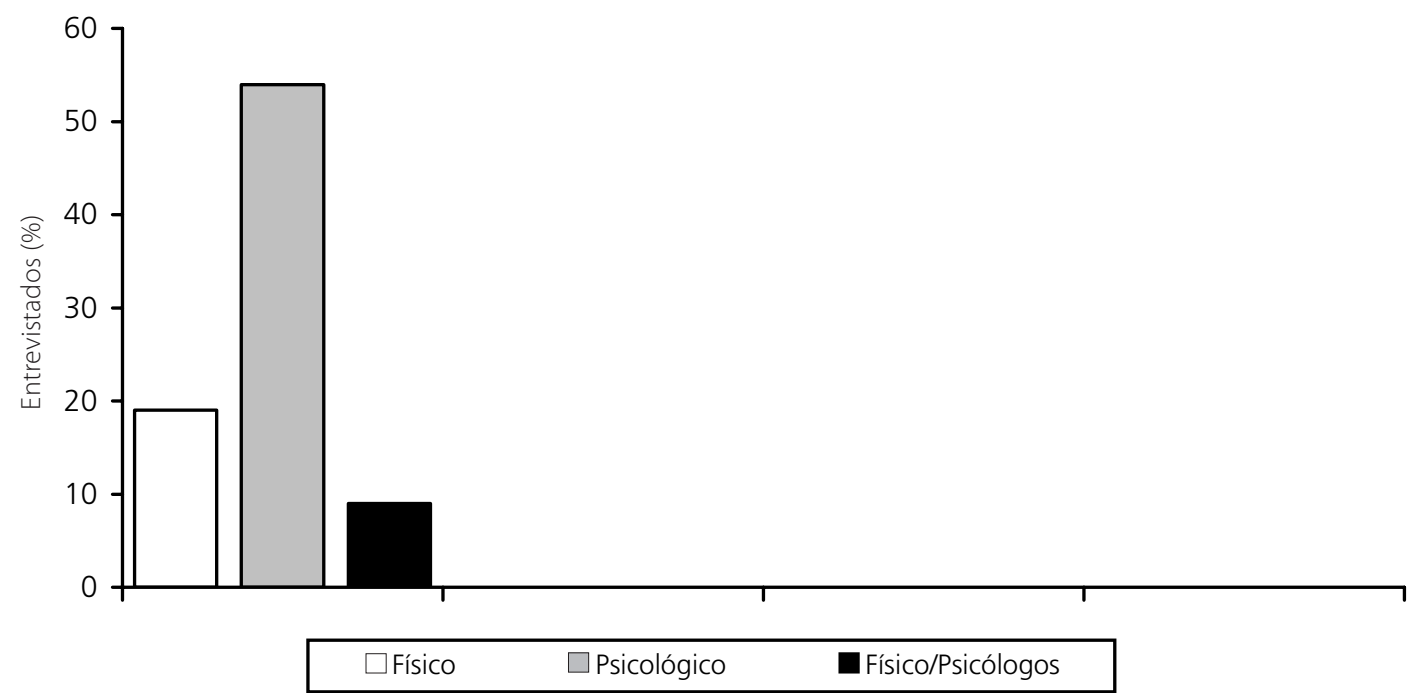

Figura 4. Distribuição da incidência de sintomas do stress entre os entrevistados da capital. 
mais verbalizados foram sensação de desgaste físico constante, mal-estar generalizado sem causa específica, cansaço constante e problemas com a memória.

\section{Discussão}

A presente pesquisa investigou a incidência do stress e sua sintomatologia em pessoas que passaram por um serviço de atendimento comunitário na capital e no interior do Estado de São Paulo. O estudo preocupou-se, também, em analisar a diferença quanto ao nível de stress entre os participantes que moravam na capital e no interior.

A amostra revela que o stress esteve presente nos dois grupos de pessoas residentes em locais diferentes. Esse dado mostra que independentemente do local de residência das pessoas, o stress pode se manifestar, embora os agentes desencadeadores do stress possam ser diferentes. $O$ estudo não avaliou as fontes de stress dos participantes; talvez esses dados possam ser coletados em outra pesquisa.

O stress tem sido pesquisado em várias populações. O estudo realizado por Lipp et al. (1996) verificou o nível de stress entre homens e mulheres na cidade de São Paulo. Participaram desse estudo 1818 pessoas de ambos os sexos, sendo que 32\% delas apresentaram sintomas significativos de stress. Verificou-se, também, uma diferença significativa entre homens e mulheres; as mulheres apresentaram mais sintomas de stress que os homens. Os dados mostraram que o stress tem afetado também a população que vive, trabalha ou circula pela cidade de São Paulo. A presente pesquisa também encontrou a presença de sintomas significativos de stress em pessoas residentes na capital.

Os participantes da pesquisa foram entrevistados durante um programa comunitário - que oferece serviços especializados gratuitos à população - promovido por uma universidade particular. Observa-se que as pessoas que usufruíram desse programa comunitário possuíam diversos níveis de escolaridade, desde o ensino fundamental até a pós-graduação. Contudo, verifica-se que os participantes do interior possuíam nível de escolaridade mais baixo, ensino fundamental, quando comparados com os da capital, nível superior. Esse dado pode indicar que independentemente da escolaridade, o stress pode estar presente, e também corrobora o estudo de Calais, Andrade e Lipp (2003). As autoras investigaram a escolaridade e o stress, e verificaram que as mulheres eram mais afetadas do que os homens em todas as faixas etárias pesquisadas.

Os participantes desempenhavam diversas funções profissionais, sendo que a maior parte estava empregada. Portanto não se pode, exclusivamente, relacionar nível de stress e área de atuação profissional, deve-se considerar outras variáveis.

A idade média entre os participantes se concentrou na terceira década, que caracteriza a fase adulta, com prevalência das pessoas do sexo feminino. Whitkin Lanoil (1985) afirma que existe uma diferença entre os sexos quanto ao stress: as mulheres possuem estressores, ou seja, o que causa stress, que é específico do gênero.

Ressaltando essa questão e analisando os resultados obtidos pelo presente trabalho, pode-se inferir que o nível de stress na fase de resistência encontrado na maioria dos entrevistados pode ter sofrido implicações específicas quanto ao sexo, pois a maioria entrevistada era do sexo feminino, tanto no interior, quanto na capital.

Quanto à incidência do stress, verificou-se que o stress em nível moderado esteve presente em mais da metade dos participantes. A fase da resistência é caracterizada por uma quebra da homeostase interna, provocando uma série de sintomas, tanto físicos quanto psicológicos. Lipp e Malagris (1995) mencionam que nessa fase existe uma tentativa do reequilíbrio interno, o que acarreta gasto de energia, e assim ocasiona uma sensação de desgaste generalizado sem causa aparente e esquecimentos. A falta de memória sinaliza que a demanda ultrapassou a capacidade da pessoa em lidar com a situação presente.

O stress elevado pode contribuir para diversas doenças. O estudo realizado por Lipp et al. (1996) avaliou o stress associado a alterações de pressão arterial na população de São Paulo, capital. Foram pesquisadas 1818 pessoas. Os resultados mostraram que 8\% dos entrevistados tinham pressão arterial acima de 140 por $90 \mathrm{mmHz}, 32 \%$ apresentaram sintomas significativos do stress e 7\% dos indivíduos com stress tinham pressão elevada. Esse estudo ressalta a importância do stress poder estar associado a problemas cardiológicos, seja como fator de risco ou desencadeador. Embora a 
presente pesquisa não tenha avaliado as implicações do stress ou associações com doenças, ressalta-se que o manejo de stress para essas pessoas poderia colaborar de modo preventivo.

Lipp (2003) menciona que quando o organismo consegue se adaptar e resistir ao estressor, o processo de stress se interrompe, e não traz prejuízos para as pessoas. No entanto, se isso não ocorre, ele avança para fases mais severas.

Observou-se que os sintomas psicológicos foram os mais mencionados. Esse dado foi também encontrado em outras pesquisas sobre stress, como Torrezan e Lipp (1996), Baechtold (2002), Cipriano (2002). Existem outras manifestações emocionais, como ansiedade e depressão, que foram pesquisadas na população da cidade de São Paulo. O estudo foi realizado por Borges e Angelotti (2002) com 30 pacientes, de ambos os sexos, que procuraram um hospital geral localizado na zona sul de São Paulo. Os resultados mostraram que $36,67 \%$ dos sujeitos encontravam-se acima da média e 16,67\% muito acima da média quanto à ansiedade. Verificou-se que 43,33\% encontravam-se acima da média e 36,67\% muito acima da média quanto à depressão. Esses dados ressaltam a importância do estudo e do aprimoramento de programas que possam atuar preventivamente, nos mais diversos transtornos emocionais, inclusive o stress.

As autoras, Lipp e Malagris (2001), mencionam os efeitos do stress na população. Referem que uma sociedade saudável e desenvolvida requer pessoas com habilidades para lidar com as situações do dia-a-dia. Porém, se o stress for excessivo na comunidade, os adultos podem se tornar frágeis e sem resistência para enfrentar os problemas e dificuldades da vida.

A avaliação do stress na população torna-se relevante uma vez que os programas de prevenção e tratamento podem se basear nessas informações para sua elaboração e planejamento. O relatório sobre saúde mental no mundo de 2001 menciona como base para um programa, a informação atualizada e idônea a respeito da comunidade, para formar os indicadores de saúde mental, os tratamentos eficazes, as estratégias de prevenção e a promoção de recursos para a saúde mental. De acordo com essa afirmação, o presente estudo colaborou com uma parcela de informações a respeito da comunidade, além de esclarecer e orientar os participantes quanto ao stress, seus sintomas e como manejá-lo. No entanto, recomenda-se que sejam elaborados programas específicos para essa população que busca atendimento comunitário.

\section{Conclusão}

O efeito do stress moderado foi encontrado na maior parte da amostra. Portanto, pode-se concluir que - stress afeta as pessoas de modo geral, independentemente do local em que residam. O que causa esse stress pode ser ou não diferente para as condições no interior ou na capital. Sugere-se que estudos sejam realizados com outras populações para verificar esse dado. Recomenda-se que programas especializados na prevenção e tratamento do stress excessivo sejam oferecidos à comunidade para que possam ajudar as pessoas a terem uma vida mais saudável.

\section{Referências}

Areias, M.E.Q., \& Guimarães, L.A.M. (2004). Gênero e estresse em trabalhadores de uma universidade pública do estado de São Paulo. Psicologia Estudos, 9 (2), 255-62.

Baechtold, A.P. (2002). Qualidade de vida, fontes internas e sintomas de stress em uma amostra de costureiras. Dissertação de mestrado, Departamento de Pós-Graduação em Psicologia, Pontifícia Universidade Católica de Campinas.

Borges, N.B., \& Angelotti, G.S. (2002). Ansiedade e depressão em uma amostra de pacientes classificados como portando fatores psicológicos que afetam as condições médicas. Estudos de Psicologia, 19 (3),15-22.

Cannon, W.B. (1953). Bodily changes in pain, hunger, fear and rage. Boston: Brandford.

Calais, S.L., Andrade, L.M.B., \& Lipp, M.E.N. (2003). Diferenças de sexo e escolaridade na manifestação do stress em adultos jovens. Psicologia: Reflexão \& Crítica, 16 (2),257-263.

Classificação Estatística Internacional de Doenças e Problemas Relacionados à Saúde CID - 10 (1997). (10a ed.). São Paulo: EDUSP.

Cipriano, D.G.I. (2002). Stress feminino: efeitos diferenciais do relacionamento afetivo. Dissertação de mestrado, Departamento de Pós-Graduação em Psicologia, Pontifícia Universidade Católica Campinas.

Everly, G., \& Rosenfeld, R. (1981). The Nature and Treatment of the stress Response. New York: Plenum Press.

Lipp, M.E.N. (1984). Stress e suas implicações. Estudos de Psicologia, 1 (3,4), 5-19.

Lipp, M.E.N. (2000). Inventário de sintomas do stress para adultos. São Paulo: Casa do Psicólogo. 
Lipp, M.E.N. (2003). O modelo quadrifásico do stress. In M.E.N. Lipp (Org.). Mecanismos neuropsicofisiológicos do stress: teorias e aplicações clínicas (pp.17-22). São Paulo: Casa do Psicólogo.

Lipp, M.E.N., Romano, A.S.P.F., Covolan, M.A., \& Silva Nery, M.J.G. (1990). Como enfrentar o stress (3a ed.). São Paulo: Ícone.

Lipp, M.E.N. et al. (1996). Diferenças em nível de stress entre homens e mulheres na cidade de São Paulo. Anais do Simpósio sobre stress e suas implicações: um encontro internacional (p.122). Campinas.

Lipp, M.E.N., Pereira, I.C., Floksztrumpf, C., Muniz, F., \& Ismael, S.C. (1996). Incidência de stress e hipertensão na população de São Paulo. Anais do simpósio sobre stress e suas implicações: um encontro internacional (p.123). Campinas.

Lipp, M.E.N., e Malagris, L.N. (1995). Manejo do stress. In B. Rangé. (Orgs.). Psicoterapia comportamental e cognitiva: teoria, pesquisa e aplicações. Campinas: Ed. Psy II.

Lipp, M.E.N., \& Malagris, L.N. (2001). O stress emocional e seu tratamento. In B. Range. Terapias cognitivo-comportamentais: um diálogo com a psiquiatria (pp.475-89). Porto Alegre: ArtMed.
Molina, O. F. (1996). Estresseno cotidiano. São Paulo: Editora Pancast.

Relatório sobre a saúde no mundo. (2001). Saúde mental: uma nova concepção, nova esperança (pp. 111-43). Organização Pan-Americana de Saúde e Organização Mundial da Saúde.

Selye, H. (1965). Stress a tensão da vida (2a ed.). São Paulo: Ibrasa.

Spielberger, C.D. (1979). Understanding stress and anxiety. London: Harper \& Kow.

Torrezan, E.A., \& Lipp, M.E.N. (1996). Incidência de sintomas de stress e de fontes estressoras em gestantes. Anais do Simpósio sobre stress e suas implicações: um encontro internacional (p.127).

Torrezan, E.A. (1997). As implicações do stress nas doenças. Revista PSICO-USF, 2 (1):13-22.

Webster, M. (1950). New word dictionary of the american language. New York: The World Publishing Company.

Witkin-Lanoil, G.A. (1985). A síndrome do stress feminino. Rio de Janeiro: Editora Imago.

Recebido para publicação em 29 de abril de 2004 aceito em 5 de janeiro de 2005. 